\title{
Discovery via Integration of Experimentation and Modeling: Three Examples for Titanium Alloys
}

\author{
Y. LIU,${ }^{1}$ P. SAMIMI,${ }^{1}$ I. GHAMARIAN,${ }^{1}$ D. A. BRICE,${ }^{1}$ D. E. HUBER ${ }^{2}$ \\ Z. WANG ${ }^{1}$ V. DIXIT, ${ }^{3}$ S. KODURI ${ }^{3}$ H. L. FRASER, ${ }^{2}$ and P. C. COLLINS ${ }^{1,4}$ \\ 1.-Department of Materials Science and Engineering and the Center for Advanced Research and \\ Testing, University of North Texas, Denton, TX 76203, USA. 2.-Department of Materials Science \\ and Engineering, The Ohio State University, Columbus, OH 43210, USA. 3.-Intel Corporation, \\ Hillsboro, OR 97006, USA. 4.-e-mail: Peter.Collins@unt.edu
}

\begin{abstract}
One key aspect of any integrated computational materials engineering approach is the integration of experiments that provide critical information for the modeling activities. This article describes, using case studies, three examples of critical experiments that have been conducted in an integrated fashion with modeling activities for titanium alloys, providing valuable information in an accelerated manner. The first has been used to identify key microstructural features associated with fracture toughness in Ti-6Al-4V and integrates artificial neural networks and various experimental techniques. The second is associated with defect accumulation in highly constrained titanium structures and integrates a highly innovative characterization technique (precession electron diffraction) and dislocation dynamics. The third is a high-throughput combinatorial technique to understand the oxidation behavior of titanium alloys and couples the experimental effort with the CALPHAD approach.
\end{abstract}

\section{INTRODUCTION}

The composition, microstructure, and defect structures of advanced nonferrous structural alloys are the predominant factors that govern the response of the material to an externally applied stimulus. For example, there will be an elasticplastic response of the material to an externally applied load. Similarly, there will be a compositional and structural evolution in response to thermal excursions. Although these responses are expected to occur, the precise details of the responses are less well understood. integrated computational materials engineering (ICME), or integrated computational materials science and engineering (ICMSE), is a strategy to integrate knowledge, represented by both computation/simulation and high-fidelity experimental databases, from across the composition-microstructure-processing-property paradigm so as to engineer a solution to a design problem. ICME strategies are increasingly being put into service and adding value ${ }^{1-4}$ However, to be effective the individual components must accurately describe materials phenomena.
For many structural materials, the precise details of materials response to external stimuli are not well understood. Often, an integrated experimental and computational approach can help elucidate these missing details. While not representing a complete ICME program, each of these integrated activities has provided knowledge that was otherwise lacking. Each of these programs is related to a different problem facing a particular class of nonferrous structural alloys-titanium-based alloys. These include efforts to understand the following: the influence of microstructure on the fracture toughness of $\alpha+\beta$-processed Ti-6Al-4V, the influence of defect populations on performance of single-phase and two-phase titanium alloys, and the role of composition on the oxidation behavior of several titanium alloys. The motivation and details of each problem are described separately, as are the conclusions. It is seen that the integration of critical and careful experiments with various modeling schemes can provide an understanding of the underlying material behavior that otherwise would not be known. 


\section{Case Study 1: Fracture Toughness in $\alpha+\beta$ Ti-6Al-4V}

\section{Motivation}

The global material response to the presence of a crack (i.e., the fracture toughness, $\boldsymbol{K}$ ) is an extremely difficult property to predict for a specific combination of microstructure and composition given the complicated interplay among sample geometry, initial crack size, the stress state at the crack tip, and the response of the microstructure to the local stress state. Composition and microstructure ultimately will influence both the material response to the presence of the crack through the local elastic-plastic response (i.e., strength/ductility), as well as crack path propagation. This problem is sufficiently complex so as to have been largely ignored. Consequently, the legacy research has typically focused on the inverse relationship often observed between the yield strength and the fracture toughness. However, owing to the difficult nature of the interpretation of the material response and the limited funding, there have been few efforts to move beyond this rudimentary observation and establish a mechanistic understanding of the influence of microstructure on the fracture of ductile multiphase materials in general and for Ti-based alloys specifically.

One approach to solving the problem is to combine artificial neural-network modeling with sophisticated characterization. This allows for consideration of both continuum and microscopic contributions, permitting an analysis of their interrelationships (e.g., a microstructural feature's contribution to the strength which is often associated with the continuum approach), as well as their distinct differences (e.g., the contribution of a microstructural feature on microcracking or void nucleation). This builds on previous efforts to model the tensile properties in both $\beta$ and $\alpha+\beta$-processed Ti-6Al- $4 \mathrm{~V}^{5,6}$ with an associated uncertainty of $\pm 2.5 \%$ error. These approaches have been based on neural-network models incorporating Bayesian statistics developed by MacKay ${ }^{7-10}$ and include accurate descriptions of the microstructural features based on rigorously developed stereological methods, as described elsewhere. ${ }^{11}$ In addition to their ability to make blind predictions, such models may be used to perform virtual experiments, where a single input parameter, such as a microstructural feature or solute content, is changed while all other inputs are kept fixed at some value (e.g., their average). These virtual experiments, so called because they might otherwise be impossible to achieve in the laboratory, given that the microstructural features are often very complex and interrelated, can be used to probe the functional dependencies of certain microstructural features on the mechanical properties. This effort has involved multiple database and model architecture, including models that only involve continuum variables (e.g., yield strength), models that only involve composition and microstructure, and models that involve both continuum and structural details.

\section{Methodologies}

A database was developed that consisted of: alloy composition, microstructure, tensile properties (yield strength and reduction in area), a factor that scales with plane strain thickness, and the fracture toughness (mixed $K_{1 \mathrm{C}}$ and $K_{\mathrm{Q}}$ ) for subsequent analysis. The functional dependencies as determined from the virtual experiments are sometimes quite complex. Therefore, to validate selected dependencies, specific samples were sectioned to characterize the microstructure associated with the fracture surface. From each selected sample, a region from the centerline (plane strain) was prepared using wire electrodischarge machining (EDM). These samples were prepared using conventional metallographic techniques and were polished through $0.05-\mu \mathrm{m}$ colloidal silica. They were imaged using the FEI Sirion scanning electron microscopy (SEM) (FEI Corporation, Hillsboro, OR) operating in backscattered mode at $15 \mathrm{kV}$ with a resolution of approximately $3.0 \mathrm{~nm}$. Selected locations of particular interest, including those exhibiting microstructural details associated with the fracture surface (e.g., microcracking, crack induced voids, and deformation structures in transformed $\beta$ ) were further analyzed using electron backscattered diffraction (EBSD) orientation microscopy techniques in a Philips XL-30 ESEM with TSL software (Philips, Amsterdam, The Netherlands).

\section{Results of Modeling}

The results show how the effects of continuum contributions and microscopic contributions may be isolated by comparing the functional dependencies obtained using different model architectures. Although the continuum variables that influence toughness include yield strength, sample geometry, crack size, and the resulting crack tip fields, for the purposes of this analysis the continuum inputs are limited to yield strength, ductility, and a factor that scales with plane strain thickness.

An analysis of the various models shows the importance of including both yield continuum and microscopic parameters, as these models consistently outperformed the models that excluded either the continuum details or the microscopic details. When all input parameters are included, the following observations are made.

First, the functional dependencies show a significant decrease in $K_{Q}$ with increasing yield strength for all models that include yield strength in addition to compositional and microstructural variables. Figure 1a-c shows three such models. This decrease is in keeping with the legacy data, and therefore, it is not a surprising observation. The relatively simple form of the decrease is analogous to the simple forms observed in previous work on the effect of 


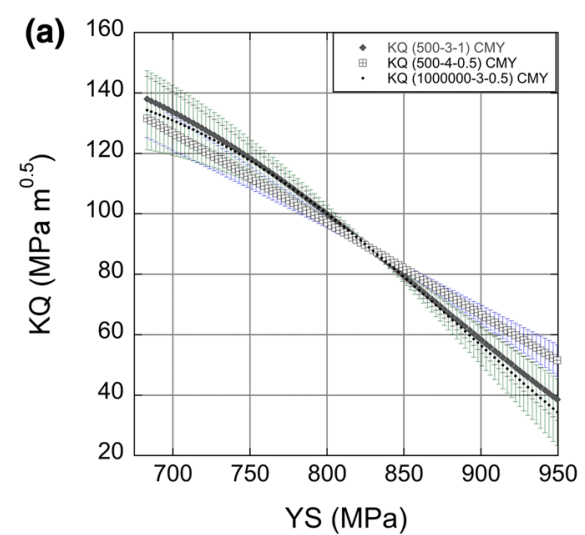

(b)
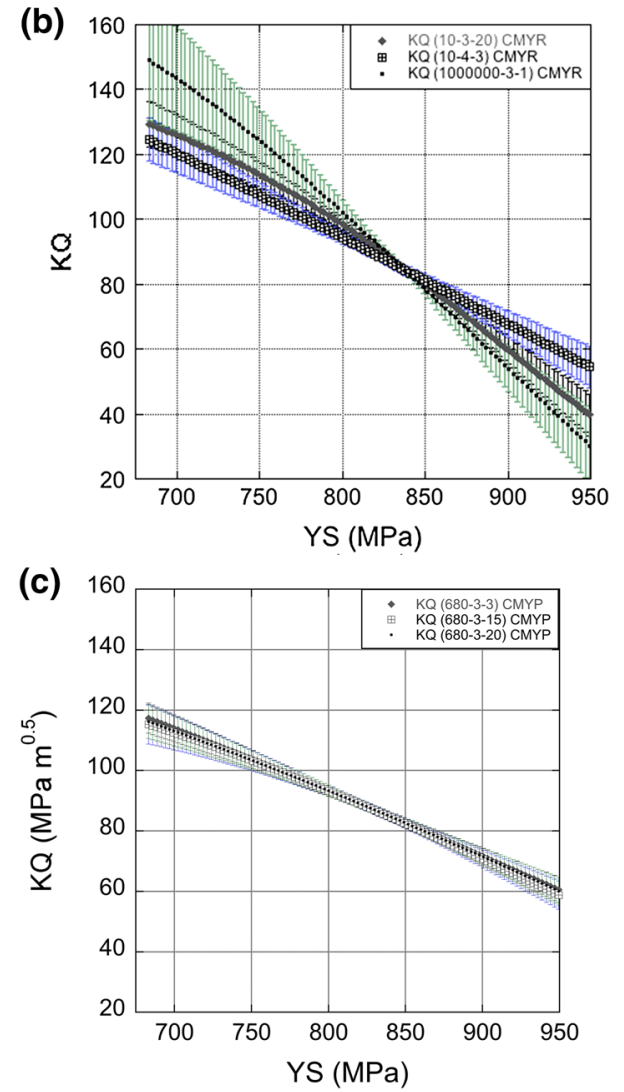

Fig. 1. Effect of $Y S$ on $K Q$ as determined by artificial neural network models which include (a) composition-microstructure-yield strength, (b) composition-microstructure-yield strength-reduction in area, and (c) composition-microstructure-yield strength-plane strain thickness.

composition or microstructure on the strength properties of $\alpha+\beta$-processed Ti-6Al-4V. It should be noted that Fig. 1c includes an estimation of plane strain thickness. When compared with Fig. 1a and $\mathrm{b}$, the slope shows a significant decrease $(\sim 40 \%)$ in the degree to which increasing yield strength decreases toughness. This result is in keeping with the fact that the database contains both $\boldsymbol{K}_{\mathbf{Q}}$ and $\boldsymbol{K}_{\mathbf{1 C}}$ data for which the state of stress influences the toughness. Consider that yield strength influences the stress state $\left(\sigma_{\mathrm{z}}\right)$ at the crack tip, and hence, whether $\boldsymbol{K}_{\mathbf{1 C}}$, a lower value, or $\boldsymbol{K}_{\mathbf{Q}}$, a higher value, is determined experimentally for a given thickness. When the plane strain thickness is included directly (Fig. 1c), the corresponding component of the influence of yield strength on toughness would diminish. Interestingly, as can be seen clearly, yield strength still has a significant effect on toughness, independent of the effect of stress state at the crack tip. The model with reduction in area included does not significantly decrease the effect of yield strength on toughness in the absence of plane strain thickness, and hence the contribution of yield strength might not be attributed solely to a stress-strain argument. Therefore, yield strength must affect the toughness in additional ways. Though the cause of the remaining influence of yield strength on toughness is not known for this two-phase, elastic-plastic, strain-hardening material, there exist three rational possibilities. First, the crack tip opening displacement is smaller for samples with higher yield strengths. It can be shown that the stress is higher for a greater distance in front of the crack tip as the radius of the crack tip decreases. Higher stresses would lead to damage accumulation in front of the crack tip (e.g., pore or crack nucleation), resulting in a lower toughness. Second, it is possible that either a microstructural or compositional variable exists that decreases the damage tolerance of the material immediately ahead of the crack tip (e.g., a reduction of the critical stress to cause pore or crack nucleation) that is inversely proportional to yield strength, yet does not influence significantly either yield strength or ductility in a negative fashion. Last, if ligament bridging occurs, then the yield strength is directly proportional to the crack tip radius and to the crack propagation. It is also quite possible that all phenomena are occurring in crack propagation in $\alpha+\beta$-processed Ti-6Al-4V.

Second, by comparing the various models, it is possible to isolate the features that influence toughness directly, and not simply by modifying the yield strength of the material. The only microstructural parameter to influence markedly toughness is the equiaxed alpha size, or a parameter that is directly proportional to the size of the equiaxed alpha particles. Similarly, iron is the only compositional variable that has a direct influence on the toughness (and yield strength).

\section{Results of Characterization}

Given that the size of the plastic zone is much larger than any microstructural feature, the size of the equiaxed alpha particles must contain a fundamental element of fracture mechanics. It turns out this is the interfacial energy. Consider that Griffith's original postulation for energy balances during fracture ${ }^{12}$ included a work term associated with the creation of surfaces of a particular energy, $\gamma_{\mathrm{s}}$. For a crack to grow, the energy for the entire system, including the work of creating new surfaces, must decrease. Therefore, rather than cleaving 

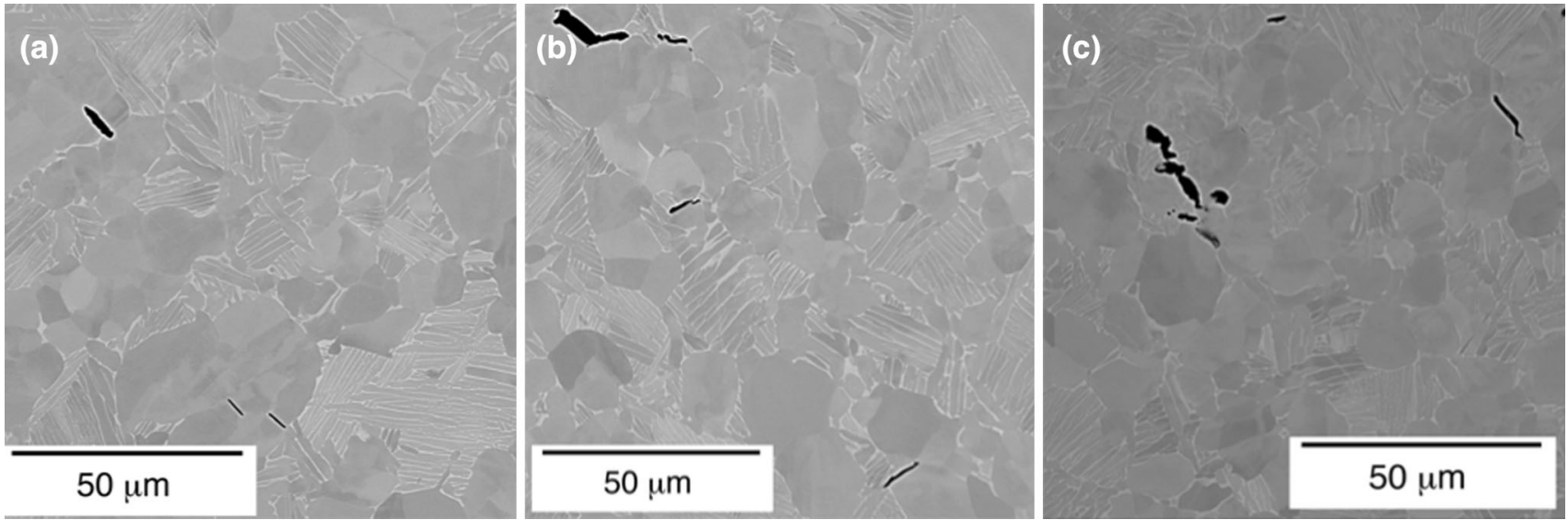

Fig. 2. (a-c) Backscattered electron micrographs showing microcracks associated with equiaxed alpha clusters.
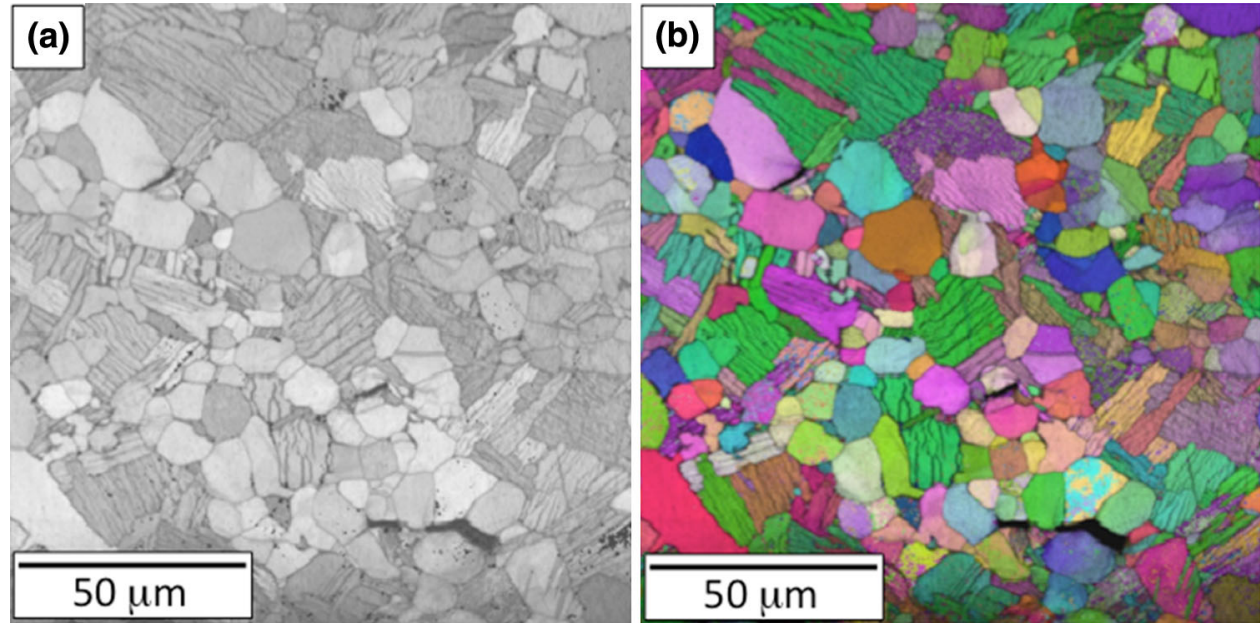

Fig. 3. Micrographs showing crack inside particle cluster using (a) orientation imaging microscopy (alpha inverse pole figure coloring with an image quality map overlaid) and (b) image quality micrograph.

through a particle (that was not observed), which would form new interfaces, cracks were expected to form at certain types of preexisting interfaces. Based on the neural networks, the equiaxed alpha particle interfaces, which are of a high energy as they do not obey Burgers orientation relationship with the beta and are thus of an arbitrary orientation and energy, are expected to crack. This was confirmed by investigating fracture specimens in a cross section.

The backscattered scanning electron micrographs shown in Fig. 2a-c are representative micrographs of subsurface microcracking that were observed frequently in many samples. The majority of the sizable cracks that were observed could be associated with the equiaxed alpha particles. Although Fig. $2 \mathrm{a}$ and $\mathrm{b}$ shows cracks in the early stage of formation or growth, Fig. 2c shows the ductile nature of fracture for a large crack within the equiaxed alpha particles. Interestingly, most of the large cracks that exist are associated with an internal structure of the equiaxed alpha particles and are not associated with the boundary between the particle and the transformed $\beta$, nor are they often associated clearly with existing $\alpha / \beta$ boundaries. The distinct contrast that is observed within the alpha particles is typically associated with channeling contrast in such backscattered electron micrographs, indicating the presence of an orientation difference and the possibility of a boundary delineating such an orientation difference. The presence of such boundaries has been confirmed using EBSD techniques. An EBSD orientation micrograph consisting of a combined alpha inverse pole figure and image quality map is shown in the backscattered SEM micrograph shown in Fig. 3a. The image quality map is shown separately in Fig. 3b. These micrographs capture the same region of interest as that shown in Fig. $2 \mathrm{~b}$, but with a $180^{\circ}$ rotation. The dark regions in Fig. $3 a$ and $b$ are associated with points where the quality of the EBSD signal is poor and hence the analysis exhibits low confidence. Often, these are associated with boundaries of some type, as in this case. It is clear from these figures 

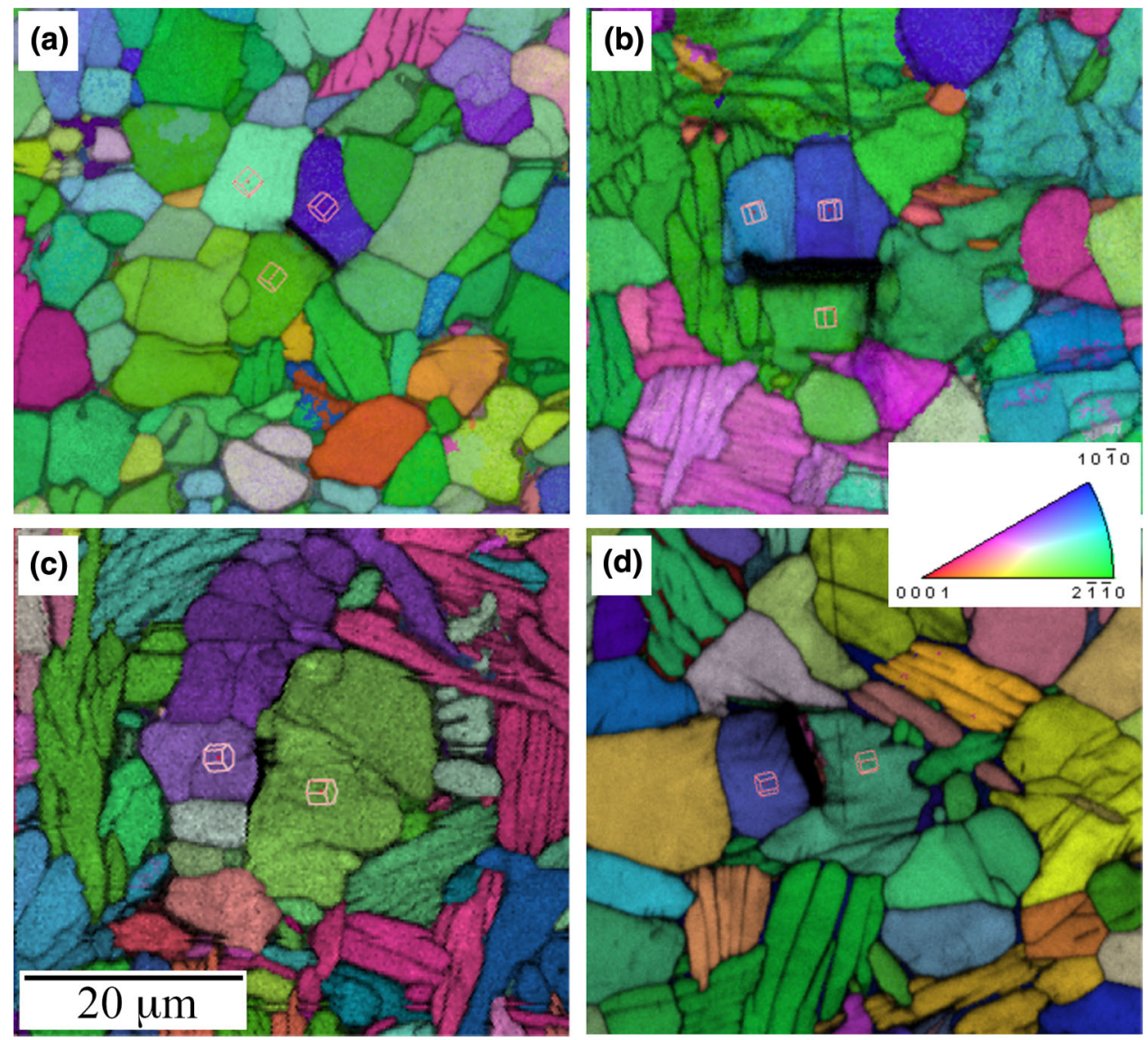

Fig. 4. EBSD maps showing cracking primarily associated with basal on basal orientations (a) $\sim 30^{\circ}$ twist along [0001], (b) $\sim 30^{\circ}$ twist along [0001], (c) $\sim 21^{\circ}$ twist along [0001], (d) 13 twist along [0001].

that the crack has followed a path delineated by misorientation, indicating the presence of boundary.

Additional EBSD analysis shows a predominance of microcracks occurring along equiaxed alpha particle surfaces between neighboring particles with nearly parallel basal planes. The microcracks appear to follow types of twist boundaries about the [0001] hexagonal close-packed (hcp) $\alpha$ zone axis. Four such examples are shown in Fig. $4 \mathrm{a}-\mathrm{d}$, with the crystal overlaid on the EBSD inverse pole figure/image quality map. Although many of these boundaries appear to occur at fairly high twist angles $\left(\sim 30^{\circ}\right.$, as in Fig. $4 \mathrm{a}$ and b, and $\sim 20^{\circ}$ in Fig. $4 \mathrm{c}$ ), some have been observed at lower twist angles $\left(\sim 13^{\circ}\right.$ in Fig. $\left.4 d\right)$. It is interesting to note that in these particles, the cracks appear to select boundaries that are nearly parallel to the basil planes. An analysis of 43 microcracks observed near eight different fracture surfaces from this data set clearly showed that 25 microcracks $(\sim 60 \%)$ were of a basil twist boundary, and 1 was a combination of basil twist/tilt. The majority of these (17 of 25) were less than $10^{\circ}$ twist from the symmetrical $30^{\circ}$ (e.g., $25^{\circ}$ to $30^{\circ}$ ) position between the [1120 ] and $[10 \overline{10}]$. This fraction $(60 \%)$ of microcracks occurring along such special boundaries is significant, as the total fraction of such boundaries in the bulk specimens has been determined to be $\sim 1.8 \%$ to $3.6 \%$. This later determination has been made from large scans of undeformed material from three specimens and calculated as a ratio of the lengths of special boundaries to length of total boundaries. Of additional interest is the fact that of the 17 microcracks that did not appear to originate from a basal twist, $7(\sim 16 \%)$ contained colony boundaries (the next highest energy interface).

The role of iron can also be explained through additional critical experimentation. There exists a subtle microstructural difference not likely to significantly affect the tensile properties, but potentially influencing microcrack formation and/or crack propagation. The difference may be observed in the backscattered electron micrographs shown in Fig. 5a and b, which are taken from two samples whose compositions are Ti-6.55Al-4.38V-0.41Fe-0.197O (Fig. 5a) and Ti-6.5Al$3.31 \mathrm{~V}-0.11 \mathrm{Fe}-0.19 \mathrm{O}$ (Fig. 5b). Clearly, the significant difference in the composition is reflected in the amount of the $\beta$-stabilizers. These two samples have nominally identical volume fraction equiaxed alpha $(67 \%$ and $66 \%$, respectively) and very similar equiaxed alpha particle sizes $(\sim 6.2 \mu \mathrm{m}$ and $\sim 6.8 \mu \mathrm{m}$, respectively). The microstructures in Fig. 5a and b corresponds with valid $K_{1 \mathrm{C}}$ 's of 70 and 51.8 , respectively. The primary 

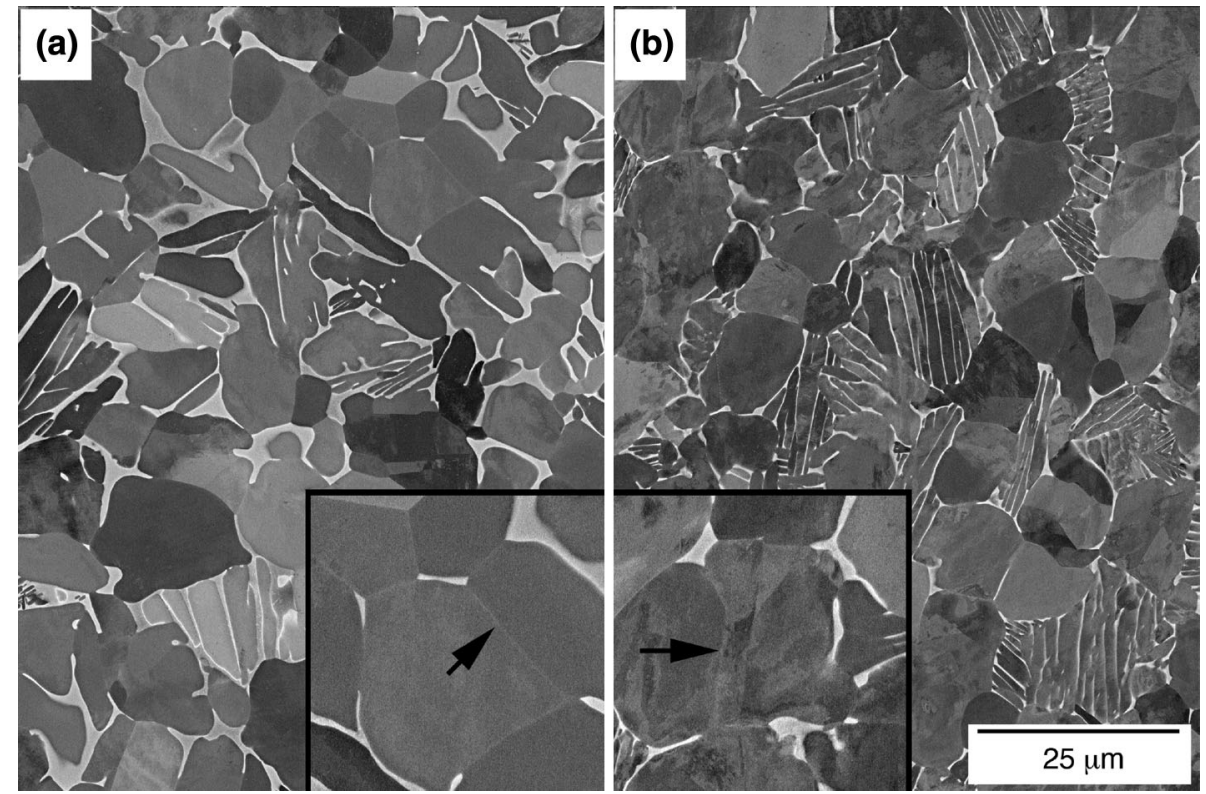

Fig. 5. (a, b) Two backscattered electron micrographs of different alloy compositions showing variation in contrast at the boundaries of the equiaxed alpha particles that is consistent with thin ribs of body-centered cubic (bcc) $\beta$ decorating the boundary in (a).

difference between these microstructures is the thickness of the $\beta$ phase separating not only the Widmanstätten $\alpha$-laths but also separating adjacent equiaxed $\alpha$-particles. The increase in the $\beta$-thickness is consistent with increasing $\beta$-stability, particularly the $\mathrm{Fe}$, which is known to be a very potent $\beta$-stabilizer. Indeed, there are extremely thin regions at the boundaries between adjacent equiaxed alpha particles whose contrast suggests the presence of beta stabilizing elements - this appears to be more predominant in Fig. 5a, consistent with the composition. The inset figures more clearly show this observation.

Based on these observations, an additional transmission electron microscopy (TEM) analysis was conducted. An undeformed portion of a sample that exhibited a $K_{1 \mathrm{C}}$ of 76.8 (Ti-4.79Al-3.35V$0.39 \mathrm{Fe}-0.196 \mathrm{O}$ ) was polished and a region defined with fiducial markers. EBSD mapping was used to identify a $30^{\circ}$ twist boundary, from which a sitespecific TEM foil was prepared using the focusedion beam (FIB). Following FIB preparation, the specimens were subjected to a final low-kV $(500 \mathrm{eV})$ $\mathrm{Ar}^{+}$milling operation using a Fischione 1040 nanomill (E. A. Fischione Instruments, Inc., Export, PA). High-resolution transmission electron microscopy was conducted on a probe corrected $300-\mathrm{kV}$ Titan TEM (FEI Company). The results reveal the two grains and the boundary (see Fig. 6a). It is clear that one grain is oriented along the $11 \overline{2} 0$ zone axis, as revealed by the fast Fourier transform (FFT) of a region of that grain (Fig. 6b). Interestingly, when a smaller region at the boundary is analyzed using FFT, the result is suggestive of a 111 body-centered cubic (bcc) zone axis (Fig. 6c). The intensity profiles shown in Fig. 6d and e further show the absence of the 0001 reflection along the boundary. In addition to these high-resolution results, the same boundary was analyzed using electron energy loss spectroscopy (EELS). The results indicate that there is a slight increase in the $\mathrm{V}$ concentration at the boundary (see Fig. $7 \mathrm{a}-\mathrm{c}$ ). In addition, there is a slight decrease in the $\mathrm{O}$ content at the boundary. These results indicate that the bcc phase is present at such equiaxed alpha boundaries, validating the SEM results.

\section{Salient Observations}

An integrated Bayesian neural-network modeling and critical experimental approach has been used to isolate the effects of composition and microstructure on the fracture toughness of $\alpha+\beta$-processed Ti-6Al$4 \mathrm{~V}$. From this integrated approach, the following conclusions are made. First, the size of the equiaxed alpha particles is the dominant microstructural feature for microstructures that fall within a certain range of microstructures. Subsequent characterization, guided by modeling, has identified a certain type of basal twist boundary as playing a key role. This boundary, occupying less than $4 \%$ of total boundary length in any specimen, can be linked with $\sim 60 \%$ of microcracking events. In addition, it has been shown that the interface plane is important; microcracking occurs when the plane is nearly parallel with the basal plane of both adjacent grains. The second most common source of microcracking is colony boundaries $(\sim 17 \%)$. The basal twist boundaries are enriched in $\beta$-stabilizers and depleted in $\alpha$-stabilizers, possibly resulting in the stabilization of the $\beta$-phase for sufficiently enriched alloys. This would explain the observation that in small amounts $(<0.41$ wt.\%), iron increases both 


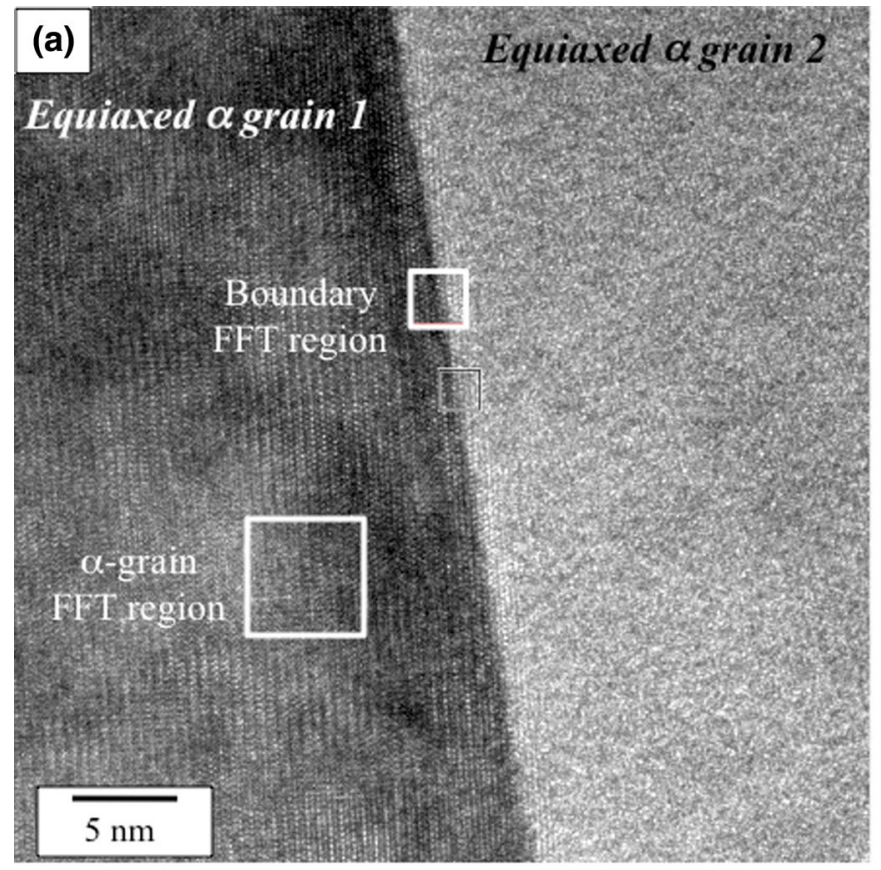

(b)

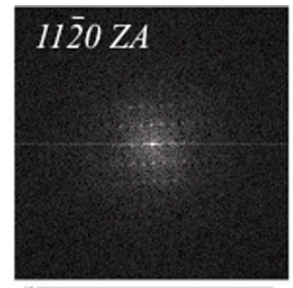

(c)

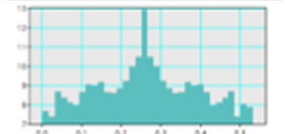

(d)

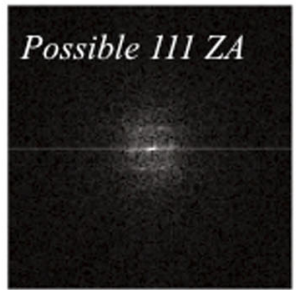

(e)

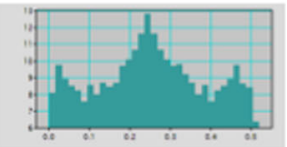

Fig. 6. (a) High resolution transmission electron micrograph showing the two grains along a boundary very close to a $30^{\circ}$ basal twist. In this region, the FFT (b) clearly shows the hexagonal close-packed (hcp) alpha phase, while the FFT and intensity profile of the FFT taken from along the boundary $(\mathrm{d}, \mathrm{e})$ suggest the possibility of local transformation to the bcc beta phase.

yield strength and toughness. The use of this combined approach has yielded many new insights into the very complex problem of fracture toughness. Indeed, such interpretations are rarely made on very ductile metallic systems such as $\alpha+\beta$-processed Ti-6Al-4V. Thus, an ulterior consequence of this approach may be its application in the development of mechanistic understandings for similarly complex properties in multiphase ductile systems.

\section{Case Study 2: Deformation Accumulation in Highly Constrained Titanium}

\section{Motivation}

In general, titanium alloys have an attractive balance of properties. However, the applications that incorporate commercially pure titanium are rather limited, owing to its relatively low strength levels. These strength levels can be increased markedly via Taylor hardening, which is possible to obtain via severe plastic deformation processes, such as the equal-channel angular pressing (ECAP) process. The ECAP process results in materials that have a significant reduction in grain size [i.e., they are ultrafine-grained (UFG) structures)] and a high density of dislocations. Many studies have been conducted on the evolution of the structure and the mechanical behavior of UFG titanium and titanium alloys. However, the data in such studies are complex to interpret, as it is not only exceptionally difficult to understand the evolution of the defect (dislocation) structures but also virtually impossible to separate the contributions of the microstructure on the evolution of the defect structure and the attending properties, given the current experimental methods. For example, the relative slip activity of a single grain is not only governed by its orientation relative to the loading axis but also governed by (and often neglected) the relative hardness of the neighboring grains and any microstructural stress concentrators that might exist. Further complicating matters in titanium alloys is the fact that, as an hcp crystal structure, anisotropic mechanical behavior is observed. Indeed, such anisotropic mechanical phenomenon has been observed for some superplastically deformed (SPD) materials with different grain sizes and texture. ${ }^{13-15}$ However, few studies have been conducted to test and understand the highly anisotropic single crystal $\alpha-\mathrm{Ti}$, whose behavior and microstructure evolutions also have very strong orientation dependence. On the one hand, this is due to the difficulty in obtaining the dislocation distribution. On the other hand, it is due to the high cost of producing large single crystals for macroscale mechanical testing.

Thus, fundamentally, we are left with a complex problem. Titanium is anisotropic and exhibits different slip activity as a function of different crystal orientation and external forces. Single-crystal experiments, while useful, are costly and can miss complex microstructural interactions. Furthermore, single-crystal experiments are generally macrolevel experiments and do not capture any potential size effects. UFG polycrystalline aggregates have been difficult to characterize, and until recently, there have not been methods to understand the defect populations. The integrated experimental and computational approaches described below are being developed to impact this complex problem. 

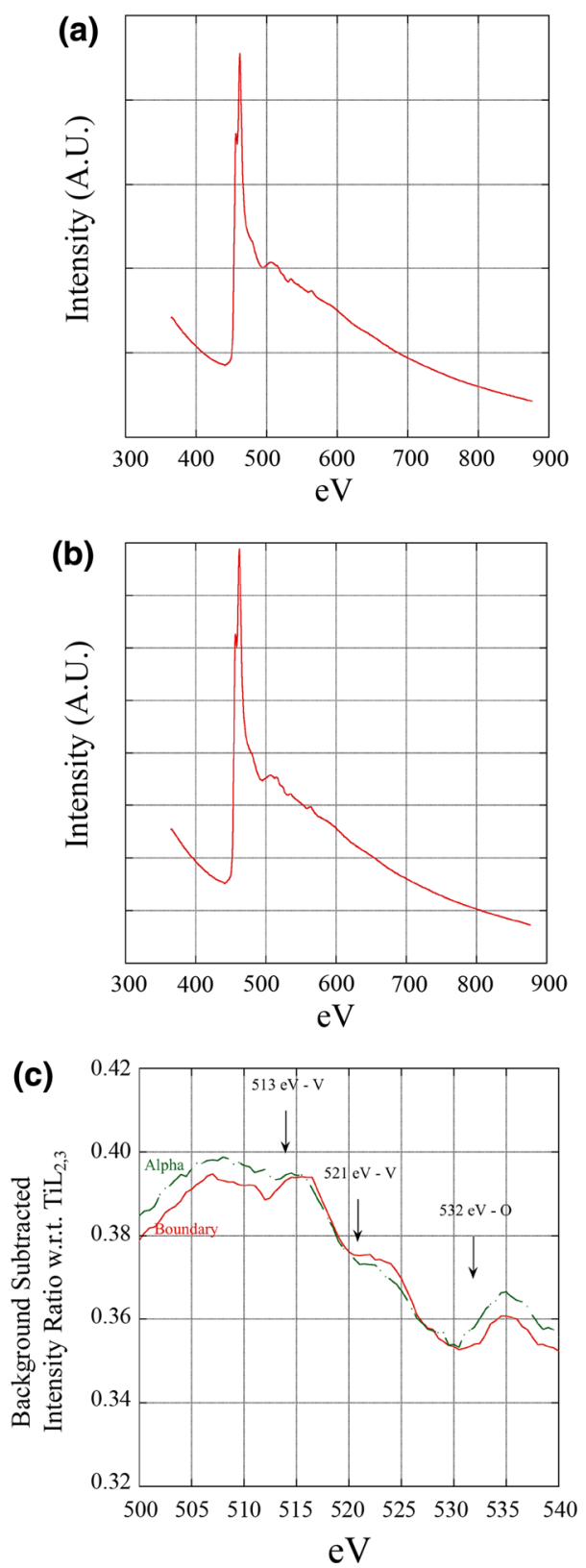

Fig. 7. EELS spectrum from (a) alpha and (b) the boundary. (c) Shows the background subtracted spectrum normalized with respect to the $\mathrm{Ti}-\mathrm{L}_{2,3}$ edge.

Once matured and integrated, it will be possible to provide key information to other levels of the ICME framework, including, for example, crystal plasticity, as will be described briefly.

\section{Methodologies}

The experimental technique relies on a relatively new method (ASTAR/precession electron diffraction (PED) from NanoMEGAS (Brussels, Belgium)) and analytical postprocessing strategies described in detail elsewhere ${ }^{16-18}$ to map out dislocation densities with a 1-nm to 3-nm resolution. As these dislocation density maps are based on the local lattice rotation as represented in the fundamental crystallographic zone, it will be possible to deconstruct these dislocation density maps and extract out each type of dislocation, thus quantifying the defect population as a function of the local crystal orientation.

The computational approach three-dimensional dislocation dynamics (3D-DD) can be used to understand the fundamental mechanisms of deformation, including dislocation structure evolution and the anisotropic mechanical behavior of materials. 3D-DD simulations, which use computers to track the motion of single and multiple dislocations, can predict microscale dislocation evolutions and link microstructure to macroscale plastic properties. Such techniques have been applied to study facecentered cubic (fcc) and bcc crystals. For example, an fcc $\mathrm{Cu}$ single crystal under high strain deformation has been simulated to understand the effect of strain rate on dislocation structure evolution. Strong anisotropic responses to deformation are observed. Both crystal orientation and strain rate affect the multiplication, glide, and annihilation of dislocations on different active slip systems, which lead to inhomogeneous substructure formation and anisotropic stress-strain curves. The formation of slip bands is found to be on the most active slip planes, depending on loading rates. Yet, the application of 3D-DD for hcp materials is limited. ${ }^{19,20}$ There are different discrete dislocation models that exist. In this work, the parametric dislocation dynamics method is used. This method uses curved segments to connect discrete dislocation nodes on a dislocation loop, which can avoid singularities in calculating dislocation self-force as the self-force is a function of dislocation line curvature. The equation of motion for a dislocation loop is

$$
\oint\left(f_{k}^{\mathrm{t}}-B_{\alpha k} V_{\alpha}\right) \partial r_{k}|\mathrm{~d} s|=0
$$

where $f_{k}^{t}=f_{\mathrm{k}}^{k}+f_{k}^{s}+f_{k}^{o}$ is the component $k$ of the total force acting on a dislocation from the summation of $f_{k}^{k}$, the Peach-Koehler force; $f_{k}^{s}$, the self-force; and $f_{k}^{o}$, the Osmotic force. $B_{\alpha k}$ is the resistive matrix, $V_{\alpha}$ is the velocity of the dislocation, and $\partial r_{k}$ is the virtual displacement of a point on the dislocation. ${ }^{21}$

To assess the influence of different structural attributes (e.g., grain size, dislocation structure, dislocation population, and loading direction), multiple cases have been computed and simulated. These include variations in grain size $(500 \mathrm{~nm}$, $1 \mu \mathrm{m}$, and $2 \mu \mathrm{m})$ and dislocation density $\left(\rho=10^{10} / \mathrm{m}^{2}\right.$, $10^{12} / \mathrm{m}^{2}$, and $10^{14} / \mathrm{m}^{2}$ ). The initial dislocation configuration consists of random distributed segments that are pinned at their ends, leading to the operation of the multiplication mechanism based on Frank-Read source activation as in real crystals. The single grain is oriented so that a tensile load is applied with a constant strain rate in a specific crystallographic direction ([0001], [11-20], and $[1-210])$. The time step is $\mathrm{d} t=5 \times 10^{12}$, and the 

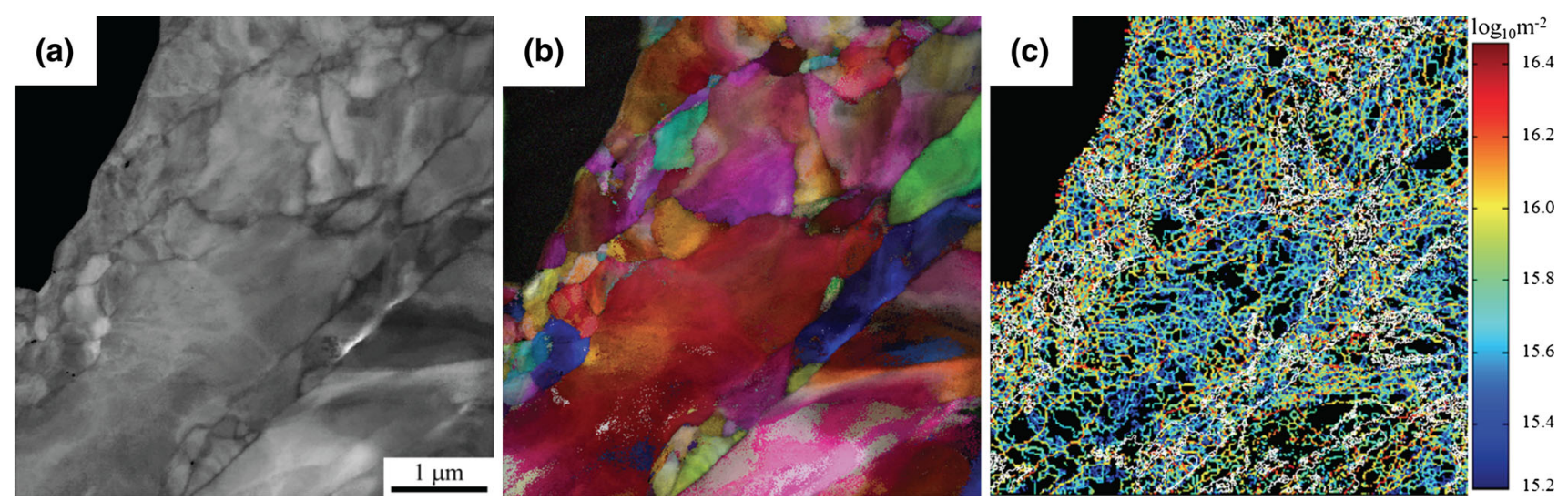

Fig. 8. Orientation results of an ultrafine grained commercially pure titanium alloy. (a) Virtual bright field image, (b) misorientation in quaternion color plot, and (c) dislocation density distribution map.

strain rate is constant at $10^{3}$ in the simulation setup. For any time $t$, the strain is given as $\varepsilon=\mathrm{d} \varepsilon /$ $\mathrm{d} t \times t .^{22}$ The lattice parameters of titanium used are $a=0.294 \mathrm{~nm}$ and $c=0.495 \mathrm{~nm}$. In these simulations, the Young's modulus $(E)$ is $130 \mathrm{GPa}$, the shear modulus $(G)$ is $40 \mathrm{GPa}$, and the numbers of dislocations range from 6 to 15 .

\section{Experimental Results}

What follows are examples of the types of results that are possible by applying the PED approach and associated algorithms for TEM-based orientation data sets. Although this methodology is very new, ${ }^{18,23-25}$ its importance and novelty cannot be overstated. It is possible to map deformation to identify dislocation arrangements via lattice rotation in highly deformed materials at a length scale of $\sim 1-2 \mathrm{~nm}$. An example of a virtual bright-field map (similar to image quality maps in EBSD) of commercially pure UFG titanium is shown in Fig. 8a, and it contains grains whose sizes range from $\sim 50 \mathrm{~nm}$ to $\sim 2 \mu \mathrm{m}$. A quaternion map, which gives a useful visualization of the orientation differences with respect to a fundamental zone is shown in Fig. 8b. Figure 8c shows a dislocation density map, where the black regions indicate a region that is largely devoid of dislocations, the white indicate grain boundaries, and the color corresponds to the dislocation density of a given data point. $^{18}$

\section{Simulation Results}

The stress-strain curves, dislocation density, and dislocation geometry are predicted as output at each simulation step. It is worth mentioning that in this simulation, the absolute values are less important than the anisotropic behavior of the material. A typical example of strain-stress curves is shown in Fig. 9a for three loading directions with the same initial dislocation density and same grain size. All curves show a linear elastic region followed by a plastic deformation. In these cases, the yield stress is defined as the point where the stress deviates from the linear elastic curve. In the meantime, an example of the dislocation density evolutions for the same three cases is shown in Fig. 9b. The densitystrain curves show that dislocation densities increase dramatically after the materials are plastically deformed, which agrees with conventional theory that dislocations are the carrier of plastic deformation.

The anisotropic behavior and grain dependence is illustrated clearly in the yield stress-grain size curve shown in Fig. 10a. As the grain size decreases, the flow stress increases as a function of grain size following the Hell-Petch mechanism, where the grain boundary acts as the barrier to the dislocation motion. The resistance to plastic deformation is due to the interactions (e.g., pile-ups) of the dislocations with the grain boundaries. Figure 10b provides the dislocation density at the 3D-DD "yield point" as a function of grain size for three loading orientations. Clearly, the evolution of dislocation density is also highly anisotropic. The geometric distribution of dislocations also can be output from 3D-DD, shown in Fig. 11a and b. Such information can be used to understand the interactions among dislocations, substructure formation, etc. The figure shows that the deformed dislocations are distributed inhomogeneously, which could be the result of anisotropic deformation as well as leading to more anisotropic deformation. Importantly, these results are consistent with the experimental maps.

\section{Salient Observations}

Both the experimental and computational results provide a method to obtain high-resolution, detailed information regarding the dislocation structures that exist in single crystals (grains) in UFG material. Both show anisotropic behavior and demonstrate that the defect populations (and consequently the response of the local grain to an external stress) are a complex 

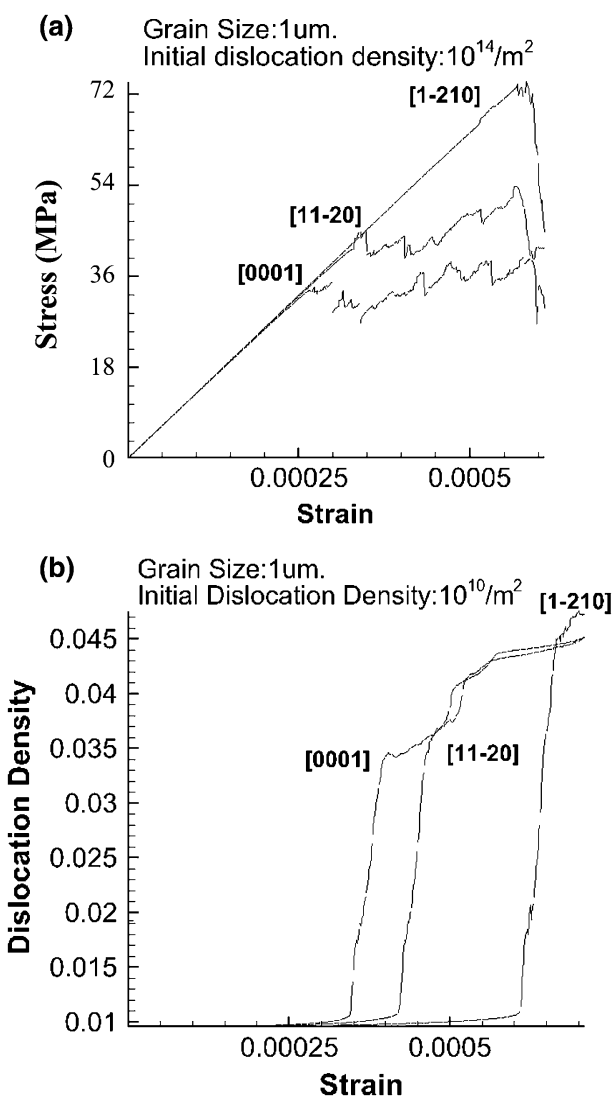

Fig. 9. Results of 3D-DD. (a) The strain-stress curves of three different loading directions. (b) The strain-dislocation density curves of three different loading directions.

problem. However, the anisotropic behavior can be analyzed qualitatively. This information, together with accurate mapping, will provide the requisite information regarding slip system hardening, which is critical for crystal plasticity calculations.

\section{Case Study 3: Oxidation Behavior of Ti-6Al-4V}

\section{Motivation}

Titanium alloys are described generally as having superior corrosion resistance at room temperature. However, at elevated temperatures, titanium suffers from poor oxidation resistance. This property limits the service temperature of titanium to $\sim 550^{\circ} \mathrm{C} .{ }^{26}$ Although the high-temperature oxidation of titanium and its alloys has been the subject of several research efforts, ${ }^{27-32}$ such studies are often constrained to the composition of specific alloys and neither the mechanisms associated with evolution of oxide layers nor the effects of oxygen on the microstructure of the material are well understood. The latter is the focus of this brief case study.

\section{Methodologies}

To understand the influence of composition on the oxidation behavior of the Ti-Mo system, a novel
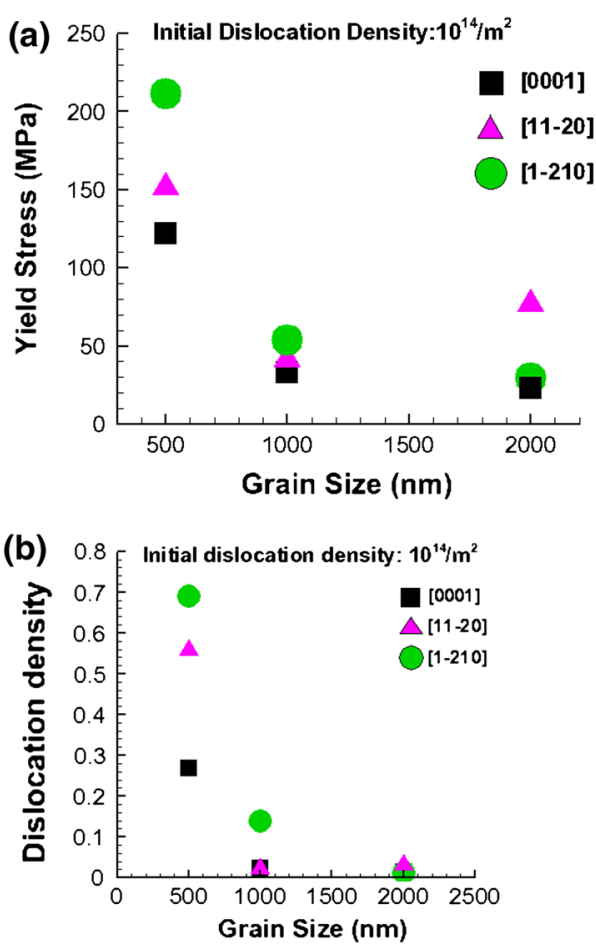

Fig. 10. 3D-DD results. (a) Yield stress plotted with respect to grain size for three loading orientations [0001], [1120 ], [1210 ] for an initial dislocation densities of $10^{14} / \mathrm{m}^{2}$. (b) Dislocation density plotted with respect to grain size and loading direction with three different initial dislocation densities.

combinatorial approach described elsewhere ${ }^{17,33,34}$ was adopted whereby a compositionally graded specimen, Ti- $x$ Mo $(0 \leq x \leq 12$, all compositions in wt.\%) was prepared using an Optomec LENS ${ }^{\mathrm{TM}}$, an additive manufacturing technique, and subsequently exposed to still air at $650^{\circ} \mathrm{C}$. Molybdenum was selected because it is an important element in high-temperature Ti alloys, such as $\beta$-21S (a $\beta$ alloy) and Ti6242 (an $\alpha+\beta$ alloy). ${ }^{31,35-39}$ This approach allows for a systematic assessment of the influence of composition on the oxidation resistance of the binary titanium alloys independent of experimental variability. The materials were $\beta$-solutionized in argon, quenched, and polished prior to being subjected to the oxidation tests. After the exposures, the specimens were prepared so that the oxide layer could be evaluated in cross section for the entire specimen. A suite of state-of-the-art characterization techniques including SEM, TEM, FIB, energydispersive spectroscopy (EDS), and atom probe tomography (APT) was employed to assess the microstructural evolution as a function of the local average composition. In addition, a thermodynamic framework based on the calculation of phase diagram (CALPHAD) approach has been coupled to rationalize all the novel microstructural transformations that have been observed. 

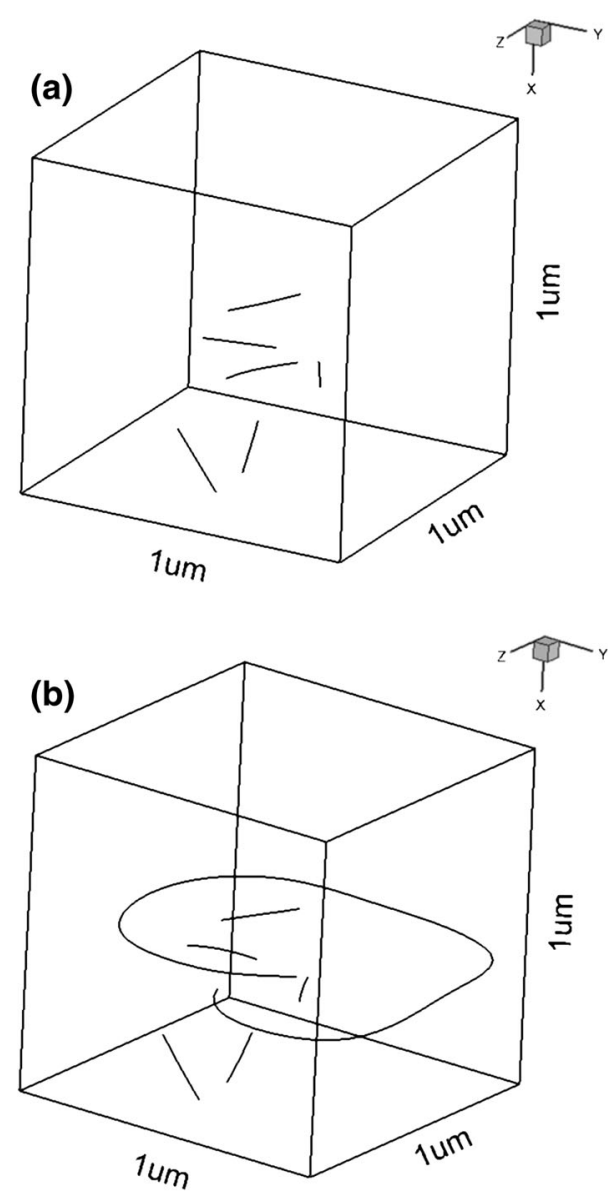

Fig. 11. 3D-DD results. (a) Initial dislocation microstructure containing straight Frank-Read sources and (b) deformed dislocation microstructure showing curved dislocations under forces.

\section{Integrated Experimental and Computational Re- sults}

A preliminary SEM analysis shows that for compositions below $\sim 8 \mathrm{wt} . \%$ Mo, the starting martensitic microstructures $\left(\alpha^{\prime}, \alpha^{\prime \prime}\right)$ have decomposed, ${ }^{40-42}$ with the bcc $\beta$ phase precipitating predominantly along prior martensitic plate boundaries or heterogeneously at the defect structures (i.e., dislocations and twins). Regardless of the variation of the phase fraction and size of the $\alpha$ laths and $\beta$ ribs, the overall phase morphologies are quite similar for compositions below $\sim 7.5$ wt. $\%$ to $\sim 8$ wt. $\%$ Mo.

Figure $12 \mathrm{a}$ and $\mathrm{b}$ shows bright-field TEM and scanning transmission electron microscopy (STEM) micrographs recorded from the outermost $3 \mu \mathrm{m}$ of the material, immediately beneath the metal/oxide interface of a Ti-3Mo component after the 25-h oxidation period. In addition to the expected $\beta$ ribs that decorate the interfaces of the prior $\alpha^{\prime}$ martensite laths, there is a surprising distribution of fine scale precipitates within the alpha laths. An elemental analysis showed that these precipitates are rich in Mo compared with the alpha phase and are consistent with compositions expected from the $\beta$-phase.
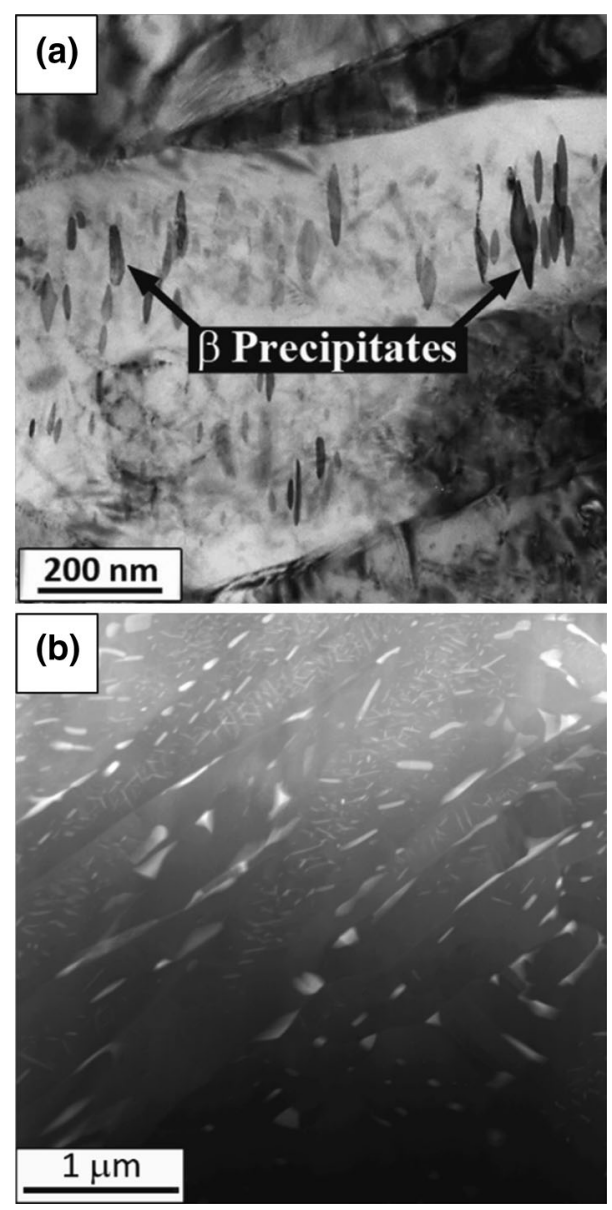

Fig. 12. (a, b) Many beam bright-field TEM micrographs of the outermost $3 \mu \mathrm{m}$ of the material, beneath metal/oxide interface of a Ti-3Mo component after the $25 \mathrm{~h}$ oxidation at $650^{\circ} \mathrm{C}$, and (c, d) STEM micrograph of the same region with Mo composition profile within a single $\alpha$ lath.

Owing to an absence of defects (e.g., dislocations), these precipitates do not appear to be associated with any interfaces of the prior $\alpha^{\prime}$ martensite laths and increase in both size and volume fraction toward the center of the $\alpha$ laths. Thus, the evidence indicates that the formation of the $\beta$ precipitates within the $\alpha$ laths is not the result of a decomposition of the martensite laths. Furthermore, as these precipitates predominately appear to be associated with $\alpha$ laths that are in direct contact with the metal/oxide interface and where the concentration of oxygen is elevated due to ingress of oxygen from the surface, oxygen plays a key role in the formation and evolution of these $\beta$ precipitates. This phase transformation, the oxygen-assisted precipitation of $\beta$ in $\alpha$, has not been reported before. TEM observations of a different composition (Ti-6 wt.\% Mo) also show this unusual precipitation of the $\beta$ phase, albeit of a larger size compared with Ti-3 wt.\% Mo (see Fig. 13).

To understand the role of oxygen in this unusual precipitation reaction, the solubility of Mo in the $\alpha$ phase has been plotted as a function of oxygen concentration under equilibrium conditions (see 


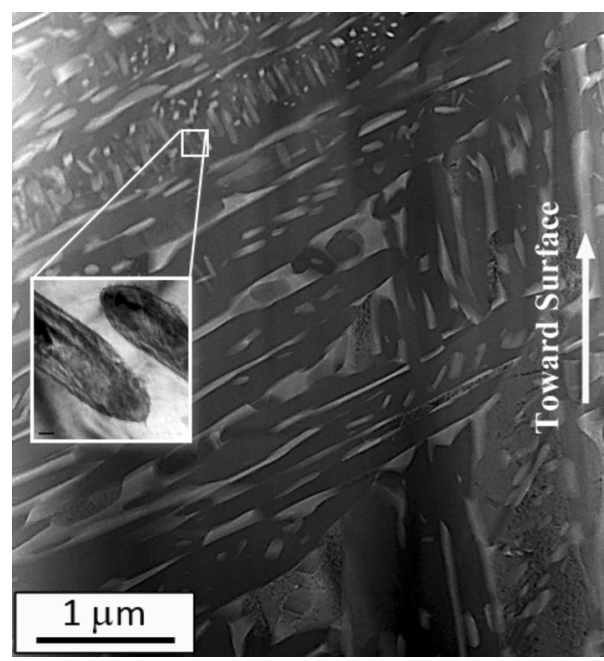

Fig. 13. STEM micrograph of the cross-section morphology of a Ti-6Mo component oxidized for $25 \mathrm{~h}$ at $650^{\circ} \mathrm{C}$.
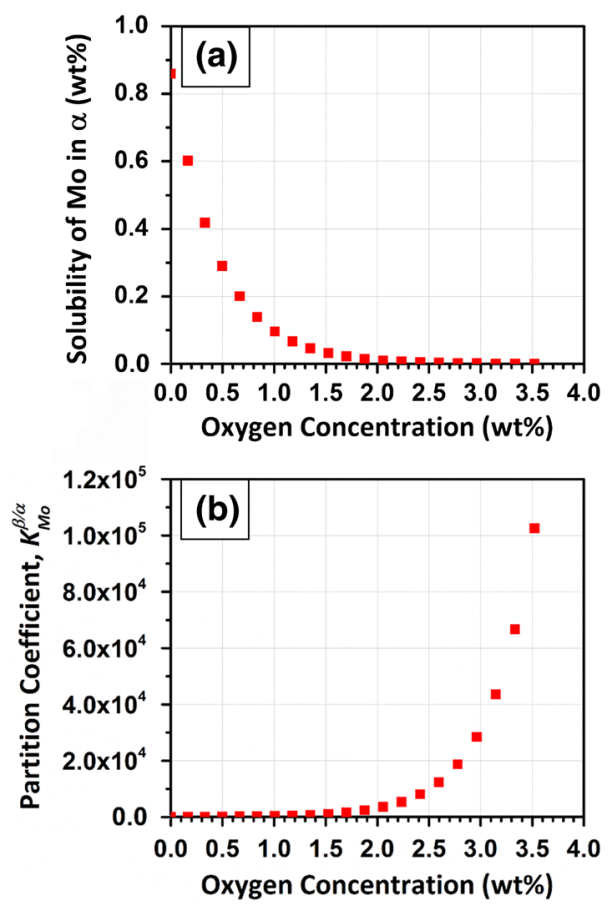

Fig. 14. (a) Solubility of Mo in $\alpha$ phase and (b) partition coefficient of Mo between the $\beta$ and $\alpha$ phases versus oxygen concentration in a Ti-3Mo component at $650^{\circ} \mathrm{C}$

Fig. 14a) using PANDAT 8.1 (CompuTherm LLC, Madison, WI), which allows for the calculation of phase equilibria of multicomponent systems ${ }^{43}$ based on the CALPHAD approach. ${ }^{44,45}$ It can be seen that Mo solubility decreases in an exponential fashion and approaches zero as the concentration of oxygen in the system increases. Thus, the diffusion of oxygen into the $\alpha$-laths during oxidation changes the distribution or partition coefficient of Mo between the $\beta$ and $\alpha$ phases $\left(K_{\mathrm{Mo}}^{\beta / \alpha}\right)$ according to Fig. $14 \mathrm{~b}$. It should be noted that there are not any obvious preferential
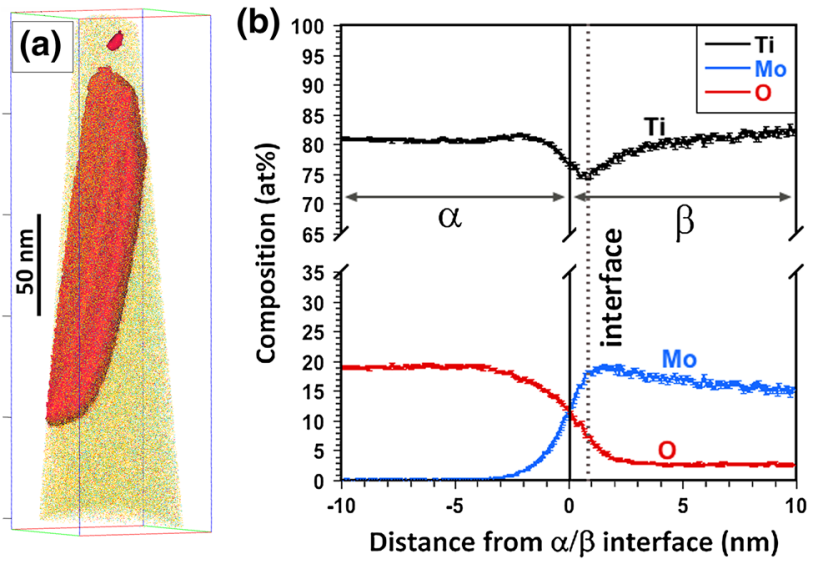

Fig. 15. (a) 3-D reconstruction of an atom probe tip created using a 9.5 at.\% Mo isoconcentration surface from the region just below the metal/oxide interface of a Ti-3Mo component after the $25 \mathrm{~h}$ oxidation at $650^{\circ} \mathrm{C}$; and (b) the corresponding proxigram that shows partitioning of elements between $\alpha$ and $\beta_{\mathrm{nb}}$.

nucleation sites for the $\beta$ precipitates that appear in the $\alpha$-laths, indicating the existence of both a barrier to the diffusion of Mo from the $\alpha$-laths to the existing $\beta$ ribs and a sufficiently large thermodynamic driving force to favor homogeneous nucleation. Considering the thickness of $\alpha$ laths and the diffusion coefficient of $\mathrm{Mo}$ in $\mathrm{Ti}^{46}{ }^{46}$ the diffusion of Mo from the alpha laths would be complete after only $\sim 15 \mathrm{~min}$. Thus, at first glance it is quite surprising that the Mo atoms do not diffuse toward the $\alpha / \beta$ interface to either precipitate a new $\beta$ rib or coarsen the existing ones. A barrier to diffusion must be present in this system. Considering the fast diffusion pathways (i.e., interfaces) for oxygen, the oxygen diffuses along the interfaces and then diffuses into the $\alpha$-laths. As oxygen enters the laths from a supersaturated interface, it changes the partitioning coefficient of Mo locally, requiring either the diffusion of Mo to the interface or towards the center of the $\alpha$-laths. The latter is favored, considering the partition coefficient and the enrichment of oxygen near the interface. Elemental analysis showed that the $\beta$ precipitates are lean in Mo relative to $\beta$ ribs, which form at the $\alpha$ lath interfaces.

To study partitioning behavior of the elements at the $\alpha / \beta$ interfaces, atom-probe tomography was employed. Figure 15a shows a 9.5 at.\% Mo isoconcentration surface for one atom-probe tip prepared from material just below the metal/oxide surface, consisting of both $\alpha$ and $\beta$ phases. The proximity histogram (i.e., proxigram) shows that from the $\alpha$ to the $\beta$ phase and across the interface, there is a decrease in the $\mathrm{O}$ concentration and an increase in the Mo to a concentration of $\sim 16$ at. $\% \mathrm{Mo}$, a concentration equivalent to the composition of the $\beta$ ribs as measured using STEM EDS. Interestingly, the partition coefficient of oxygen between the phases $\left(K_{o}^{\alpha / \beta}\right)$ varies as a function of the overall oxygen content. This was observed when the results shown in Fig. 15 (from the region just below the metal/oxide interface) 


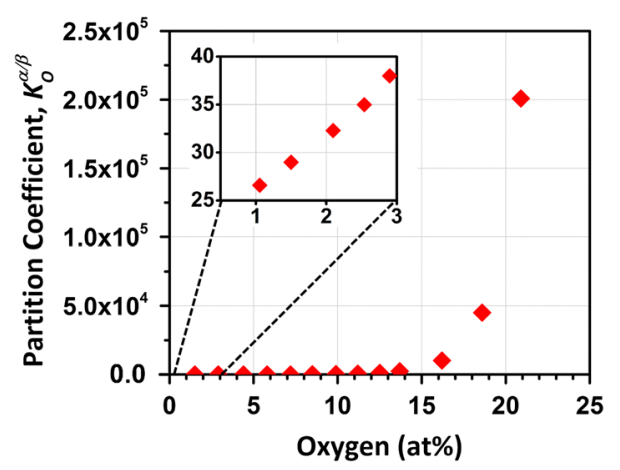

Fig. 16. The partition coefficient of $O$ between $\alpha$ and $\beta$ as a function of overall $\mathrm{O}$ content for a Ti-3Mo component at $650^{\circ} \mathrm{C}$.

were compared with another atom-probe tip extracted from a region $\sim 16 \mu \mathrm{m}$ away from the surface, and thus in a region with lower oxygen concentrations. There is a pronounced influence of bulk oxygen concentration on the oxygen content in the $\alpha$ phase and to a lesser degree in the $\beta$ phase.

Figure 16 shows the variation of $K_{0}^{\alpha / \beta}$ versus oxygen concentration under equilibrium conditions (generated using PANDAT 8.1) that leads to the abovementioned changes in the oxygen content. According to Fig. 16, for the overall composition of 2 at. $\%, K_{0}^{\alpha / \beta}$ has the value of $\sim 32$, whereas direct APT observations for the same composition showed that $K_{\mathrm{o}}^{\alpha / \beta}$ is $\sim 1$. This difference indicates that highly accurate and systematic experiments should be conducted to revisit the thermodynamic database of the Ti-Mo-O system used in this CALPHAD model.

An assessment of the Gibbs free energy-composition curves for the binary Ti-Mo and quasi-binary (Ti-1 wt.\% O)-(Mo-1 wt.\% O) at $650^{\circ} \mathrm{C}$ is shown in Figs. $17 \mathrm{a}$ and $b$, respectively. In addition to the decreasing solubility of Mo in the $\alpha$ phase and the increased $K_{\mathrm{Mo}}^{\beta / \alpha}$, there is also an observable reduction in the Gibbs energies of both $\alpha$ and $\beta$ phases. This is a significant difference that only increases upon the addition of Mo. Importantly, the change in the Gibbs free energy $\Delta G_{V}$ for the precipitation of $\beta$ from $\alpha$ is $-3 \mathrm{~kJ} / \mathrm{mol}$, a number that far exceeds the interfacial energies associated with $\alpha / \beta$ interfaces in titanium alloys, which are typically $<1 \mathrm{~J} / \mathrm{m}^{2}$. Thus, at a certain supersaturation of Mo within the lath interiors, homogeneous nucleation of $\beta$-precipitates occurs within the $\alpha$-laths.

\section{Salient Observations}

Both the experimental and computational results demonstrate how the ingress of oxygen changes the phase stabilities and can drive unexpected microstructural evolutions in titanium alloys at regions immediately beneath the oxide layer. These stabilities, which were not previously established experimentally, can be understood by invoking thermodynamic modeling strategies, and some details of the databases need to be refined. Although the databases require refinement to improve the partitioning coefficient details, it is
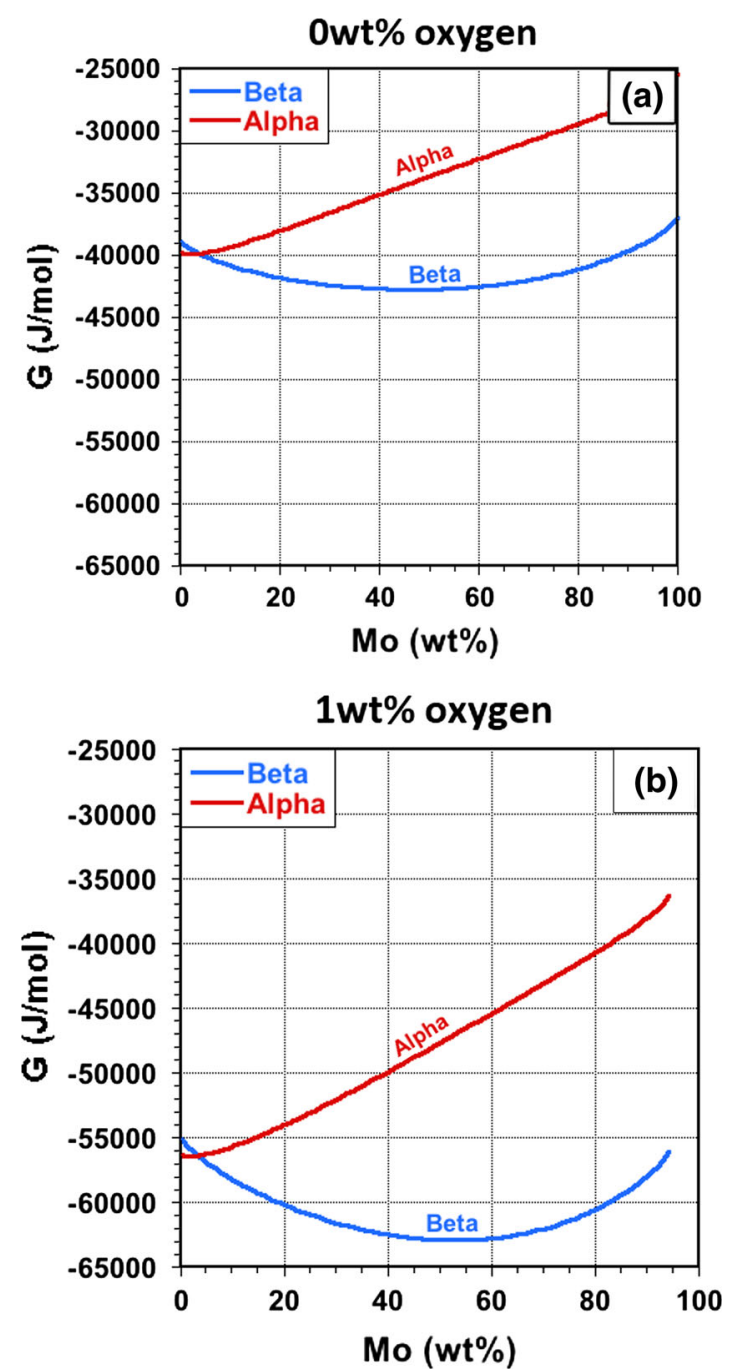

Fig. 17. Gibbs free energy versus composition for the (a) binary Ti-Mo and (b) quasi-binary (Ti-1 wt.\% O)-(Mo-1 wt.\% O) at $650^{\circ} \mathrm{C}$.

expected that the CALPHAD method would allow for the prediction of the influence of alloying elements on the formation of these microstructural features in the near-surface region by calculating both the partition coefficients and the $\Delta G_{V}$ for the precipitation of $\beta$ from $\alpha$.

\section{CONCLUSION}

Three research activities associated with the influence of composition and microstructure on the properties of titanium alloys have been presented. In each activity, the integrated experimental and computational approach has been instrumental in identifying mechanisms or details of the problem that were previously unknown. The three activities are motivated by three distinctly different problems, namely: (I) understanding what governs fracture toughness in $\alpha+\beta$-processed Ti-6Al-4V, (2II) how deformation proceeds in ultrafine grained titanium, and (III) the influence of alloy composition on the oxidation behavior of titanium alloys. The conclusions of each are summarized below: 
- Fracture toughness of $\alpha+\beta$-processed Ti-6Al-4V: Through an integrated modeling and critical experimental approach, it has been possible to deduce that one microstructural feature directly influences the fracture toughness. This feature, i.e., the size of the equiaxed alpha particles, influences not only yield strength but also fracture toughness. The size is related to the probability that a specific boundary-namely a basal twist boundary-is present in the microstructure. This boundary, when present, will result in microcracking ahead of the crack tip resulting in lower fracture toughness. This interface can be further modified by alloying with increased levels of $\beta$-stabilizers, such as $\mathrm{Fe}$, which may positively influence the fracture toughness behavior.

- Deformation of ultrafine grained titanium: The integrated approach confirms the anisotropic nature of deformation in these materials and provides the first quantitative details of the dislocations that are present in deformed UFG materials. Ultimately, it is expected that this information will be extended providing dislocation-level information including the type of dislocation, which can vary in hcp materials. The next steps will include considering nearest-neighbor grain orientations and dislocation density content.

- Influence of alloy composition on oxidation behavior of titanium alloys: Although this effort was initiated as a high-throughput combinatorial approach, the observations in many respects were surprising, including the precipitation of $\beta$ from the $\alpha$ phase. To understand these phase transformations, a series of calculations was conducted using the CALPHAD method and resulted in an understanding of the thermodynamic factors that contribute to these observations, especially the changes in solubility and partitioning behavior. Now that these features have been observed, and are expected to have an impact on the near-surface mechanical properties, the CALPHAD approach can be used not only to predict similar observations in other Ti-based systems but also to assess whether ternary alloying elements would impact, for example, the partition coefficient of the $\beta$-stabilizers.

\section{ACKNOWLEDGEMENTS}

The authors would like to acknowledge the support provided by the Office of Naval Research, program \# 2626753 Direct-3-Dimensional (D-3D), Dr. Julie Christodoulou, program officer. The authors also gratefully acknowledge the University of North Texas; the Air Force Research Laboratory; the Center for Advanced Non-Ferrous Structural Alloys, which is a joint industry-university center between the Colorado School of Mines and the University of North Texas (NSF award Number 1134873); and the Center for Advanced Research and Testing at the University of North Texas.
The authors would also like to thank Dr. Babu Viswanathan for invaluable technical discussions.

\section{OPEN ACCESS}

This article is distributed under the terms of the Creative Commons Attribution License which permits any use, distribution, and reproduction in any medium, provided the original author(s) and the source are credited.

\section{REFERENCES}

1. J. Allison, JOM 63 (4), 15 (2011).

2. J. Allison, M. Li, C. Wolverton, and X. Su, JOM 58 (11), 28 (2006).

3. P. Collins, C. Haden, I. Ghamarian, B. Hayes, T. Ales, G. Penso, V. Dixit, and G. Harlow, JOM 66, 1299 (2014).

4. B.A. Cowles and D. Backman, Advancement and Implementation of Integrated Computational Materials Engineering (ICME) for Aerospace Applications, Tolland: DTIC Document (OH: Wright-Patterson Air Force Base, 2010).

5. P. Collins, S. Kart, T. Searles, S. Koduri, G. Viswanathan, J. Tiley, R. Banerjee, and H. Fraser, Frontiers in the Design of Materials (FDM-NMD-ATM), ed. R. Baldev, S. Ranganathan, S.L. Mannan, K. Bhanu Sankara Rao, M.D. Matthew, and P. Shankar (Boca Raton, FL: CRC Press, 2007), p. 19.

6. S. Kar, T. Searles, E. Lee, G. Viswanathan, H. Fraser, J. Tiley, and R. Banerjee, Metall. Mater. Trans. A 37, 559 (2006).

7. D.J. Mackay (Ph.D. dissertation, California Institute of Technology, 1992)

8. D.J. MacKay, Neural Comput. 4, 448 (1992).

9. P.C. Collins, R. Banerjee, and H.L. Fraser, Scr. Mater. 48, 1445 (2003)

10. P.C. Collins, S. Koduri, B. Welk, J. Tiley, and H.L. Fraser, Metall. Mater. Trans. A 44, 1441 (2013).

11. P.C. Collins, B. Welk, T. Searles, J. Tiley, J.C. Russ, and H.L. Fraser, Mater. Sci. Eng. A 508, 174 (2009).

12. A.A. Griffith, Philos. Trans. R. Soc. London A 221, 163 (1921)

13. K.S. Kumar, H. Van Swygenhoven, and S. Suresh, Acta Mater. 51, 5743 (2003).

14. G.G. Yapici, I. Karaman, and H.J. Maier, Mater. Sci. Eng. A 434, 294 (2006).

15. S. Nemat-Nasser, W. Guo, and J. Cheng, Acta Mater. 47, 3705 (1999).

16. I. Ghamarian, Y. Liu, P. Samimi, and P.C. Collins, unpublished work (2014).

17. P. Samimi, Y. Liu, I. Ghamarian, J. Song, and P. Collins, Acta Mater. 69, 92 (2014).

18. I. Ghamarian, Y. Liu, P. Samimi, and P.C. Collins, Acta Mater. 79, 203 (2014).

19. G. Monnet, B. Devincre, and L.P. Kubin, Acta Mater. 52, 4317 (2004).

20. L. Capolungo, I. Beyerlein, and Z.Q. Wang, Model. Simul. Mater. Sci. Eng. 18, 085002 (2010).

21. N. Ghoniem, S.-H. Tong, and L. Sun, Phys. Rev. A 61, 913 (2000).

22. S. Wright and R. Larsen, J. Microsc. 205, 245 (2002).

23. E. Rauch, K. Barmak, J. Ganesh, P. Ferreira, A. Darbal, D. Choi, T. Sun, B. Yao, K. Coffey, and S. Nicolopoulos, Microsc. Microanal. 17, 1086 (2011).

24. E.F. Rauch, J. Portillo, S. Nicolopoulos, D. Bultreys, S. Rouvimov, and P. Moeck, Z. Kristallogr. 225, 103 (2010).

25. H. Mohseni, P. Collins, and T. Scharf, Nanomater. Energ. 1, 318 (2012)

26. G. Lütjering and J.C. Williams, Titanium (New York: Springer, 2007).

27. H. Siegel, R. Duncan Jr., and R. Swift, Scaling of Titanium and Titanium Alloys (Lexington, KY: Kentucky Research Foundation, 1955). 
28. M. Mitoraj, E. Godlewska, O. Heintz, N. Geoffroy, S. Fontana, and S. Chevalier, Intermetallics 19, 39 (2011).

29. J. Hickman and E. Gulbransen, Anal. Chem. 20, 158 (1948).

30. T.A. Wallace, R.K. Clark, and K.E. Wiedemann, Oxidation Characteristics of Beta-21S in Air in the Temperature Range 600 to $800 \mathrm{C}$ (Hampton, VA: National Aeronautics and Space Administration. Langley Research Center, 1992).

31. H. Garbacz and M. Lewandowska, Mater. Chem. Phys. 81, 542 (2003).

32. R. Padma, K. Ramkumar, and M. Satyam, J. Mater. Sci. 23, 1591 (1988).

33. P.C. Collins, R. Banerjee, S. Banerjee, and H.L. Fraser, Mater. Sci. Eng. A 352, 118 (2003).

34. R. Banerjee, D. Bhattacharyya, P.C. Collins, G.B. Viswanathan, and H.L. Fraser, Acta Mater. 52, 377 (2004).

35. A. Ebach-Stahl, C. Eilers, N. Laska, and R. Braun, Surf. Coat. Technol. 223, 24 (2013).

36. R. Shenoy, J. Unnam, and R. Clark, Oxid. Met. 26, 105 (1986).

37. R.K. Clark, J. Unnam, and K. Widemann, Oxid. Met. 28, 391 (1987).
38. T.A. Wallace, R.K. Clark, and K.E. Wiedemann, NASA Technical Memorandum, 104228 (1992).

39. G. Xu, G. Yang, J. Zhu, and Y. Peng, Rare Met. 18, 176 (1999).

40. R. Davis, H. Flower, and D. West, J. Mater. Sci. 14, 712 (1979).

41. H.M. Flower, R. Davis, and D.R.F. West, Titanium and Titanium Alloys, ed. J.C. Williams and A.F. Belov (New York: Springer, 1982), p. 1703.

42. G. Welsch, R. Boyer, and E.W. Collings, Materials Properties Handbook: Titanium Alloys (Materials Park, OH: ASM International, 1993).

43. L. CompuTherm, CompuTherm LLC, Madison, WI 53719 (2008).

44. L. Kaufman and H. Bernstein, Computer Calculation of Phase Diagrams with Special Reference to Refractory Metals (New York: Academic Press, 1970).

45. N. Saunders and A.P. Miodownik, CALPHAD (Calculation of Phase Diagrams): A Comprehensive Guide: A Comprehensive Guide (Atlanta, GA: Elsevier Science, 1998).

46. S. Malinov, W. Sha, and P. Markovsky, J. Alloys Compd. 348, 110 (2003). 